\title{
Chronic glucolipotoxic conditions in pancreatic islets impair insulin secretion due to dysregulated calcium dynamics, glucose responsiveness and mitochondrial activity
}

\author{
Baggavalli P Somesh ${ }^{\dagger}$, Mahesh Kumar Verma ${ }^{\dagger}$, Manoj Kumar Sadasivuni ${ }^{\dagger}$, Anup Mammen-Oommen ${ }^{\dagger}$, \\ Sanghamitra Biswas, Pavagada C Shilpa, Ashok Kumar Reddy, Aggunda N Yateesh, Puttrevana M Pallavi, \\ Siddaraju Nethra, Rachapalli Smitha, Korrapati Neelima, Usha Narayanan and Madanahalli R Jagannath*
}

\begin{abstract}
Background: In the progression towards diabetes, glucolipotoxicity is one of the main causes of pancreatic beta cell pathology. The aim of this study was to examine the in vitro effects of chronic glucolipotoxic conditions on cellular responses in pancreatic islets, including glucose and fat metabolism, Calcium mobilization, insulin secretion and insulin content.

Results: Exposure of islets to chronic glucolipotoxic conditions decreased glucose stimulated insulin secretion in vitro. Reduced protein levels of Glut2/slc2a2, and decreased glucokinase and pyruvate carboxylase mRNA levels indicated a significant lowering in glucose sensing. Concomitantly, both fatty acid uptake and triglyceride accumulation increased significantly while fatty acid oxidation decreased. This general suppression in glucose metabolism correlated well with a decrease in mitochondrial number and activity, reduction in cellular ATP content and dampening of the TCA cycle. Further, we also observed a decrease in IP3 levels and lower Calcium mobilization in response to glucose. Importantly, chronic glucolipotoxic conditions in vitro decreased insulin gene expression, insulin content, insulin granule docking (to the plasma membrane) and insulin secretion.
\end{abstract}

Conclusions: Our results present an integrated view of the effects of chronic glucolipotoxic conditions on known and novel signaling events, in vitro, that results in reduced glucose responsiveness and insulin secretion.

Keywords: Type 2 diabetes, Rat islets, Glucolipotoxicity, Glucose metabolism, Insulin content, Insulin secretion

\section{Background}

Type 2 diabetes mellitus (T2DM) is a metabolic disorder in which pancreatic insulin secretion does not meet the demands of insulin sensitivity [1,2]. Over a period of time, consistently elevated levels of blood glucose and free fatty acids lead to glucolipotoxicity- mediated pancreatic beta cell dysfunction $[3,4]$. It is now accepted that elevated glucose levels are required to mediate the lipotoxic effects, including inhibition of glucose-stimulated insulin secretion (GSIS), impaired insulin gene expression and apoptosis [4-8].

\footnotetext{
*Correspondence: m.r.jagannath@connexios.com

${ }^{\dagger}$ Equal contributors

Connexios Life Sciences Pvt Ltd., No. 49, First Main road, 3rd phase, JP Nagar, Bangalore 560 078, India
}

GSIS involves both glucose oxidation-coupled ATP production and the anaplerotic/cataplerotic pathway-mediated generation of coupling factors that trigger and amplify insulin secretion, respectively $[9,10]$. Briefly, glucose uptake initiates metabolic pathways in which glucose is first converted to pyruvate mediated by glucokinase, and then to oxaloacetate by pyruvate carboxylase. Mitochondrial oxaloacetate generates citrate, a cataplerotic signal, which is transported to the cytosol and then broken down into acetyl-CoA initiating fatty acid synthesis. Acetyl-CoA is subsequently converted to malonyl-CoA, the concomitant step in fatty acid synthesis. In pancreatic beta cells, malonyl-CoA inhibits carnitine-palmitoyl transferase-1 (CPT-1) blocking fatty acid oxidation and resulting in the buildup of long- 
chain acyl-CoA esters (LC-CoA) in the cytosol [10]. Long chain-CoA is thought to be a potential modulator of insulin secretion stimulating insulin granule docking and exocytosis [11,12]. Glucose metabolism also raises the cytosolic ATP/ADP ratio, which inhibits the ATP-sensitive potassium channel $\left(\mathrm{K}_{\mathrm{ATP}}\right)$ resulting in plasma membrane depolarization. In response to this, voltage-gated calcium channels open, causing an influx of extracellular calcium and exocytosis of insulin granules [13].

Another well-known role of glucose is augmenting insulin secretion by promoting phospholipase-C (PLC)-mediated hydrolysis of phosphatidylinositol 4, 5-biphosphate (PIP2) into diacylglycerol (DAG) and inositol triphosphate (IP3) [14]. The DAG generated, in turn, activates protein kinase $C$ (PKC), which is known to maintain insulin exocytosis $[15,16]$, while IP3 mobilizes calcium from endoplasmic reticulum stores. The PLC pathway is also known to upregulate cAMP levels in beta cells, which show glucosemediated oscillations that correlate with insulin secretion $[17,18]$.

Further, glucose is known to increase insulin content through insulin gene transcription mediated by PDX1 and MAFa [19]. Under normal conditions, the synthesized insulin is held in readily releasable pools which are transported to the plasma membrane by the small GTPase, Rab27a and the SNARE complex for acute calcium-mediated release [20,21].

Chronic hyperglycemia (glucotoxicity) and hyperlipidemia (lipotoxicity) have been known to impair beta cell function [22,23], and glucolipotoxicity has been defined as 'the deleterious effects of elevated glucose and fatty acids on pancreatic beta cell-function and mass' [24]. Studies by Kashyap et al. in human subjects have shown that the ability of the beta cell to increase insulin secretion in response to fatty acids is a component that may predispose to T2DM [25]. In accordance with this, animal models for T2DM show a glucolipotoxicity-mediated dysfunction in multiple cellular processes involved in insulin secretion [26-27 and references therein]. In vitro studies have been an important source of information to understand the molecular basis of glucolipotoxicity. For example, fatty acid-mediated inhibition of insulin gene transcription, which was identified in vitro, has been recapitulated in vivo. However, a known limitation of the in vitro studies in this area of research has been the varying concentrations of fatty acid used [26].

Here, we used specific concentrations of glucose and palmitate to study the effects of in vitro chronic glucolipotoxic conditions on intracellular signaling pathways and cellular processes that mediate glucose responsiveness and insulin secretion. We confirmed metabolic stress in pancreatic islets under these conditions using known stress markers. We found that chronic glucolipotoxicity impaired glucose and fat uptake/metabolism in rat pancreatic cells resulting in lower cellular ATP along with mitochondrial number and activity. In agreement with this, IP3 levels were also reduced as was the calcium mobilized by the IP3 receptor and the L-type voltage gated calcium channels. Finally, we found that chronic glucolipotoxicity significantly decreased insulin secretion by reducing both insulin gene expression and granule docking to the plasma membrane in pancreatic islets. Thus, our results present the first integrated view of glucolipotoxicity in vitro linking known and novel signaling events to reduced glucose sensitivity and insulin secretion.

\section{Results}

To investigate the effects of chronic glucolipotoxicity on glucose responsiveness and insulin secretion, we generated glucolipotoxic conditions in rat pancreatic islets and the NIT1 beta cell line using $16.7 \mathrm{mM}$ glucose and $500 \mu \mathrm{M}$ palmitate.

\section{Chronic glucolipotoxicity reduces insulin secretion in rat pancreatic islets}

To evaluate the effect of high glucose and fatty acid concentrations on insulin secretion, we incubated rat pancreatic islets as mentzioned above for $72 \mathrm{~h}$ (chronic glucolipotoxic conditions that mimic diabetic pathology [26]); untreated islets were used as control. Under these conditions, we treated rat pancreatic islets with either low glucose or high glucose for $2 \mathrm{~h}$ to study glucose-stimulated insulin secretion (GSIS) (Figure 1). In agreement with previous studies [27], in the presence of high glucose, islet insulin secretion was significantly reduced under chronic glucolipotoxic conditions (Figure 1). We confirmed induction of glucolipotoxicitymediated ER stress, oxidative stress and inflammation in pancreatic islets using known metabolic stress markers $[28,29]$ (Additional file 1: Figure S1 and Additional file 2: Figure S2).

To understand the mechanism by which chronic glucolipotoxic conditions reduce GSIS in vitro, we next

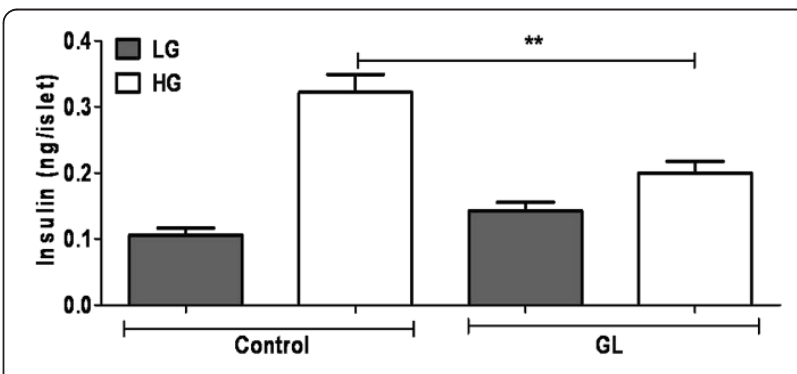

Figure 1 Chronic glucolipotoxic conditions in vitro reduce GSIS. Rat islets were cultured under normal conditions (control) or with $16.7 \mathrm{mM}$ glucose and $500 \mu \mathrm{M}$ palmitate for $72 \mathrm{~h}$ (GL). Post $72 \mathrm{~h}$, islets were treated with $2 \mathrm{mM}$ glucose (LG) or $11 \mathrm{mM}$ glucose (HG) for $2 \mathrm{~h}$ and secreted insulin was measured. Data are expressed as mean \pm SEM and statistical analysis was performed using the unpaired Student's t-test. ${ }^{*} \mathrm{P}<0.05,{ }^{* *} \mathrm{P}<0.01$ and $\left.{ }^{*}{ }^{*} \mathrm{P}<0.001, \mathrm{n}=4\right)$. 
assessed glucose uptake/metabolism, calcium release, insulin gene expression and granule docking.

\section{Glucose uptake and metabolism is impaired under chronic glucolipotoxic conditions}

We examined the effect of chronic glucolipotoxic conditions on glucose metabolism in rat pancreatic islets and NIT1 cells maintaining the same experimental conditions used in Figure 1. Untreated rat islets and NIT1 cells, respectively were used as controls. We found significant reductions in the mRNA and protein levels of the glucose transporter, Glut2/Slc2a2 under chronic glucolipotoxic conditions when compared to the untreated control (Figure 2A and B) [30]. This reduction in Glut2 seen in both NIT-1 cells and rat pancreatic islets (Figure 2B) suggested impaired glucose metabolism, which was confirmed by a decrease in Glucokinase (Gck) and pyruvate carboxylase (Pc) mRNA levels (Figure 2C and E) [31-33]. To determine whether glucose uptake was also affected under chronic glucolipotoxic conditions, we used a fluorescent glucose analog, 2-NBDG to monitor glucose uptake. Under chronic glucolipotoxic conditions, we observed a $\sim 40 \%$ decrease in glucose uptake (Figure 2D) indicating that both glucose uptake and metabolism were impaired. Consistent with these data, NADPH levels decreased and lactate release increased under chronic glucolipotoxic conditions (Additional file 3: Figure S3) confirming a dysfunction in glucose metabolism. The increase in lactate release also suggests that pryuvate, the end product of glycolysis, was converted into a nonoxidative metabolite indicating that glucose oxidation is severely impacted under chronic glucolipotoxic conditions.

We next ascertained the link between malonyl-CoA formation and insulin secretion under chronic glucolipotoxic conditions. To this end, we treated rat islets cultured in glucolipotoxic conditions with high glucose and found a decrease in insulin secretion, as expected. Interestingly, when ATP citrate lyase (ACLY) was inhibited using radicicol [34], insulin secretion decreased further suggesting that ACLY and potentially the anaplerotic/cataplerotic pathways are involved in the dysregulation seen in insulin secretion (Figure 2F). Together, these results suggest that chronic glucolipotoxicity impairs glucose uptake and metabolism and thus, insulin secretion.

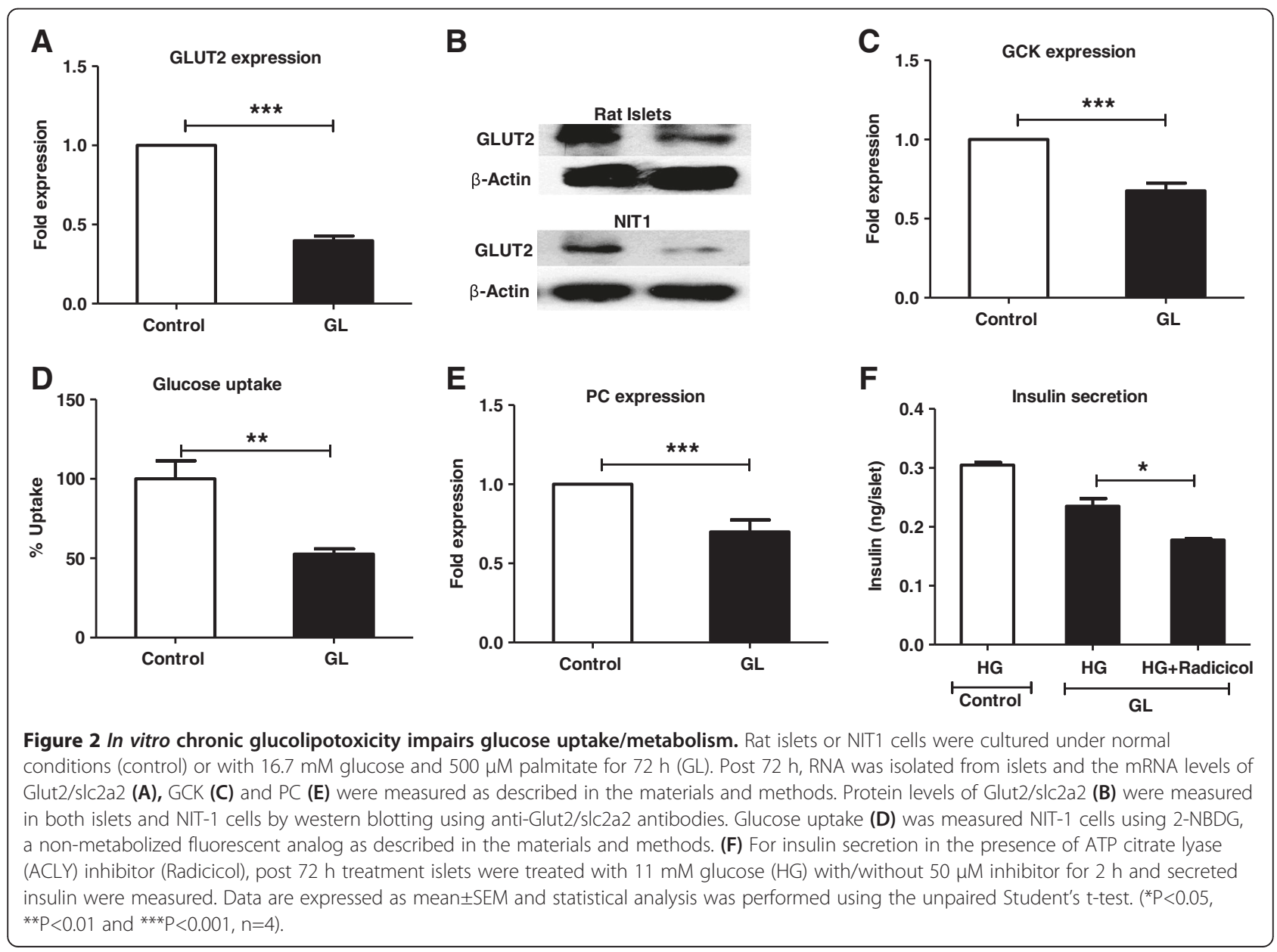




\section{Chronic glucolipotoxicity impairs fatty acid uptake and metabolism}

Since chronic glucolipotoxic conditions impaired GSIS, we next investigated its effect on fatty acid metabolism [35]. We found that mRNA and protein levels of the fatty acid transporter, cd36 were significantly increased in rat islets (Figure 3A and B). This increase was observed in both NIT-1 cells and rat pancreatic islets (Figure 3B) suggesting increased fatty acid uptake.

To ascertain whether fatty acid uptake is impaired under chronic glucolipotoxic conditions, we used a BODIPY dye, a non-metabolized fluorescently labelled fatty acid analog. We observed a three-fold increase in fatty acid uptake under chronic glucolipotoxic conditions (Figure 3C) indicating that along with CD36 mRNA and protein levels, fatty acid uptake was also impaired. Further, we also found fat metabolism to be impaired under chronic glucolipotoxic conditions as seen from the four-fold increase in triglyceride levels in the pancreatic beta cell line, NIT-1 (Figure 3D). This was validated by a reduction in fatty acid oxidation studied by measuring the mRNA levels of PPARa (Figure 3E). We confirmed that in vitro chronic glucolipotoxicity generated metabolic stress in the cell system using known markers of ER stress [28,29] (Additional file 2: Figure S2A). Taken together, these data showed that chronic glucolipotoxic conditions impaired both glucose and fatty acid uptake and metabolism.

\section{Mitochondrial number/activity and cytosolic ATP levels are reduced under chronic glucolipotoxic conditions}

Since a primary outcome of glucose metabolism is ATP synthesis from mitochondria [27], we investigated the effect of chronic glucolipotoxic conditions on mitochondrial DNA copy number/activity and cellular ATP. Under chronic glucolipotoxic conditions, mtCox 1 levels were significantly reduced in rat pancreatic islets along with a decrease in glucose-mediated cellular ATP (Figure 4A and B) suggesting a reduction in mitochondrial number. To ascertain the impact of decreased mtCox1 copy number on mitochondrial function under chronic glucolipotoxic conditions, we measured activity of succinate dehydrogenasea critical enzyme in both the citric acid cycle and the mitochondrial respiratory chain. We found that under chronic glucolipotoxic conditions, succinate dehydrogenase activity decreased by $\sim 50 \%$ (Figure $4 \mathrm{C}$ ). This reduction in mitochondrial activity was further studied by measuring insulin secretion in the presence of leucine and glutamine,

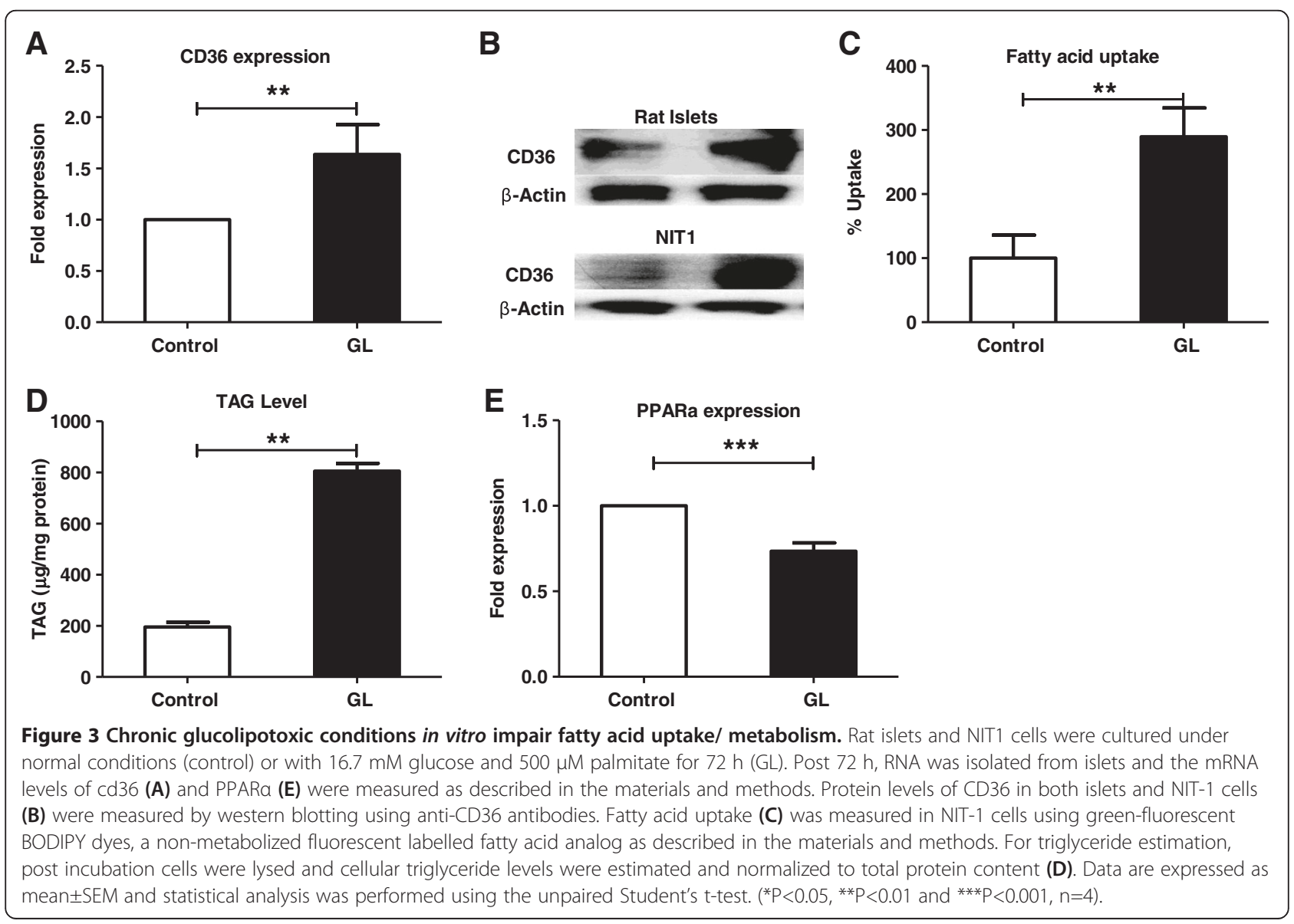



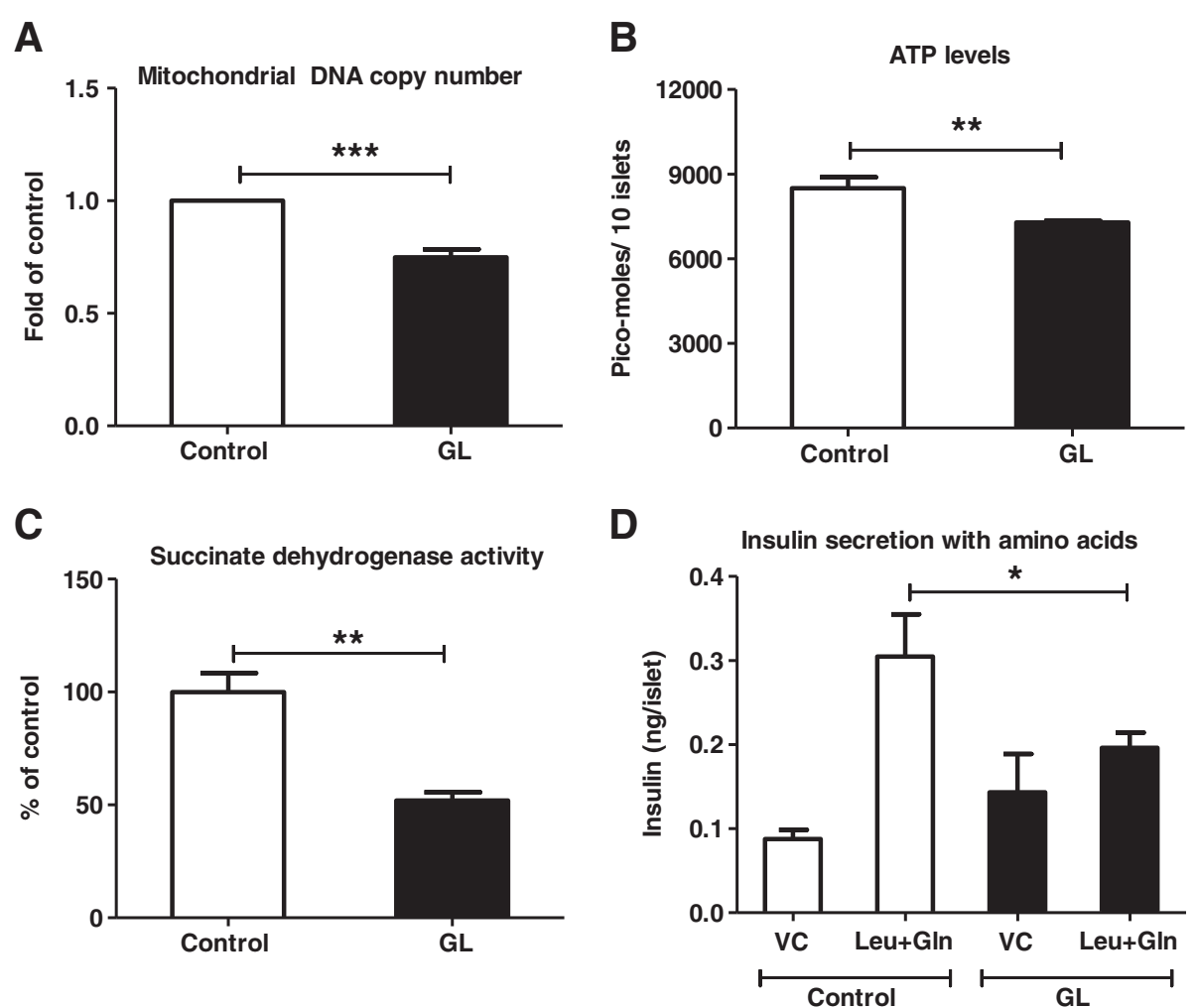

Figure 4 Chronic glucolipotoxicity in vitro reduces mitochondrial number and activity. Rat islets and NIT1 cells were cultured under normal conditions (control) or with $16.7 \mathrm{mM}$ glucose and $500 \mu \mathrm{M}$ palmitate for $72 \mathrm{~h}$ (GL). Post $72 \mathrm{~h}$, total DNA was isolated from islets and mitochondrial DNA copy number (A) was estimated by assessing mtCox1 (mitochondrial gene) copy number normalized to HPRT (nuclear gene). Post treatment, islet ATP levels (B) were estimated after 60min induction with $11 \mathrm{mM}(\mathrm{HG})$ as described in the materials and methods. Both SDH activity in NIT-1 cells (C) and amino acids ( $5 \mathrm{mM}$ of leucine and $5 \mathrm{mM}$ of glutamine) mediated insulin secretion (D) in islets were measured as described in the materials and methods. VC is vehicle control with $2 \mathrm{mM}$ glucose and without amino acids. Data are expressed as mean \pm SEM and statistical analysis was performed using the unpaired Student's t-test. $\left({ }^{*} P<0.05,{ }^{*} P<0.01\right.$ and $\left.{ }^{* * *} P<0.001, n=4\right)$.

precursors of TCA cycle intermediates [10,36]. In this assay, chronic glucolipotoxic conditions reduced insulin secretion indicating an overall suppression of the TCA cycle (Figure 4D).

These data present the first line of evidence linking a decrease in cellular ATP to a reduction in mitochondrial number and activity under chronic glucolipotoxic conditions.

An increase in cytoplasmic calcium is required for insulin secretion under chronic glucolipotoxic conditions

Since chronic glucolipotoxicity lowered GSIS and glucose metabolism, we investigated its effects on IP3 and cytosolic calcium, known signaling mediators of insulin secretion [13,17]. We detected a modest decrease in IP3 upon culturing rat pancreatic islets under chronic glucolipotoxic conditions (Figure 5A). Next, we investigated intracellular calcium dynamics under chronic glucolipotoxic conditions in NIT-1 cells; cells cultured in $5 \mathrm{mM}$ glucose were used as control. Subsequently, cells were treated with either low or high glucose and cytosolic calcium was measured. In control cells, high glucose enhanced cytosolic calcium mobilization when compared to the low glucose treatment. Interestingly, this effect of high glucose on cytoplasmic calcium was lost under glucolipotoxic conditions (Figure 5B and C).

As further confirmation, we ascertained whether L-type voltage gated calcium channels mobilized calcium under glucolipotoxic conditions by studying insulin secretion in the presence or absence of the L-type channel inhibitor, Nitrendipine, NTD [37]. As reported earlier, we detected a decrease in high glucosemediated secretion in the presence of NTD (Additional file 4: Figure S4A). In a similar assay, upon using the IP3 receptor inhibitor, 2-aminoethyldiphenyl borate (2-APB) [38], we found that endoplasmic reticulum calcium mobilization was also required for insulin secretion (Additional file 4: S4B). In summary, chronic glucolipotoxic conditions impaired IP3 levels and cytosolic calcium release. 

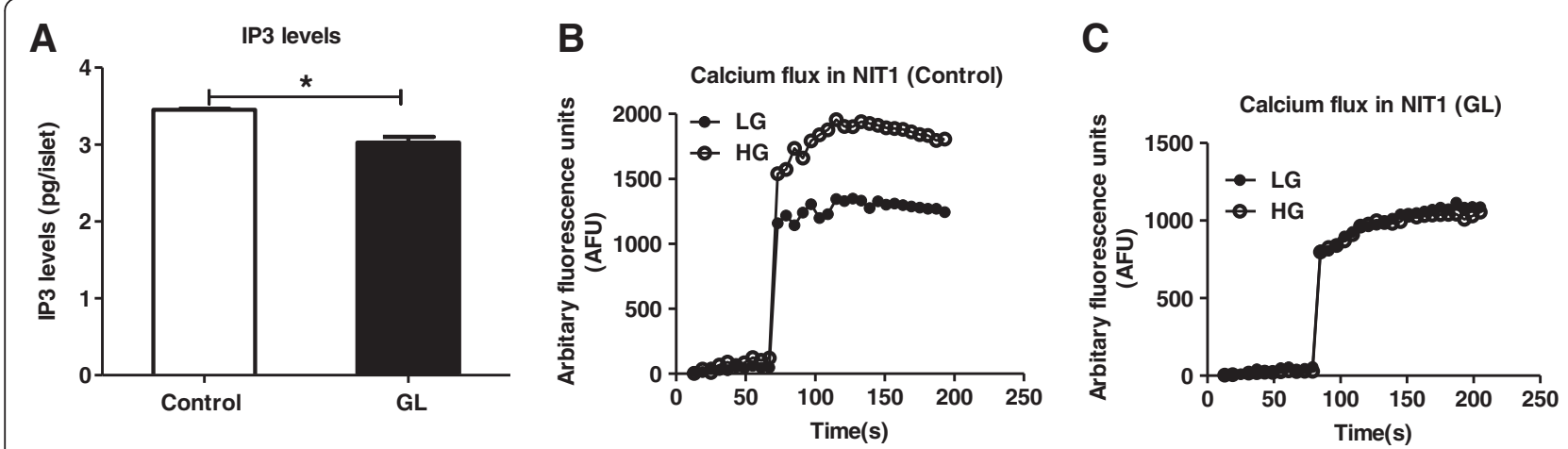

Figure $\mathbf{5}$ Chronic glucolipotoxicity in vitro impairs calcium release. Rat islets and NIT-1 cells were cultured under normal conditions (control) or with $16.7 \mathrm{mM}$ glucose and $500 \mu \mathrm{M}$ palmitate for $72 \mathrm{~h}$ (GL). Post $72 \mathrm{~h}$, islet IP3 levels (A) were estimated in the presence of $11 \mathrm{mM}(\mathrm{HG})$ as described in the materials and methods. For cytosolic calcium mobilization assay (B and C), post treatment NIT-1 cells were loaded with Fluo-3AM calcium indicator dye for $1 \mathrm{~h}$. After washing the cells, they were treated with either low (2.5 mM) or high ( $16.7 \mathrm{mM})$ glucose and the change in fluorescence was estimated as described in the materials and methods. Data are expressed as mean \pm SEM and statistical analysis was performed using the unpaired Student's t-test. ${ }^{*} P<0.05,{ }^{* *} P<0.01$ and $\left.{ }^{* * *} P<0.001, n=4\right)$.

Insulin synthesis and intracellular insulin content are reduced under chronic glucolipotoxic conditions Calcium and cAMP are known to influence insulin gene expression through Pdx1 [39,40]. As reported previously [19], Pdx1 and insulin (Ins2) mRNA levels were reduced under chronic glucolipotoxic conditions (Additional file 5: Figure S5A and B). Thus, chronic glucolipotoxicity not only mediates its effects on GSIS by impairing glucose metabolism but also a down-regulation of insulin gene transcription. Lastly, we ascertained whether changes in insulin synthesis and glucose metabolism influenced insulin content (Figure 6). To test this, we treated rat islets (as in Figure 1) and observed a significant decrease in islet insulin content under chronic glucolipotoxic conditions (Figure 6). Thus, chronic glucolipotoxicity significantly affects overall glucose responsiveness through glucose metabolism, calcium release and insulin gene expression.

\section{Insulin granule docking is reduced under chronic glucolipotoxic conditions}

In animal models of T2DM, the small GTPase, Rab27a is known to be downregulated leading to decreased insulin granule docking to the plasma membrane, thereby lowering insulin secretion [41]. In addition, insulin release at the fusion pore is also known to be impaired in diabetic animal models resulting in defects in exocytosis and release [42].

Here, we found that Rab27a mRNA significantly decreased under chronic glucolipotoxic conditions in both pancreatic islets and NIT1 cells (Figure 7A and Additional file 5: Figure $\mathrm{S} 5 \mathrm{C}$ ) suggesting a decrease in the readily releasable pool of insulin granules docked to the plasma membrane. To confirm this, we treated rat islets with potassium chloride $(\mathrm{KCl})$, a membrane-depolarizing agent known to release insulin vesicles docked to the plasma membrane [43]. As expected, we found a significant decrease in docked insulin granules under chronic glucolipotoxic conditions when compared to control islets (Figure 7B). These data show that chronic glucolipotoxicity reduces insulin secretion through its effects on insulin synthesis and transport in addition to glucose uptake/metabolism and cystosolic calcium mobilization.

Taken together, these data provide the first, integrated in vitro view of known dysfunctional cellular mechanisms in chronic glucolipotoxic conditions, while identifying novel events such as the glucolipotoxicity-mediated reduction in mitochondrial number/activity and insulin granule docking/ transport.

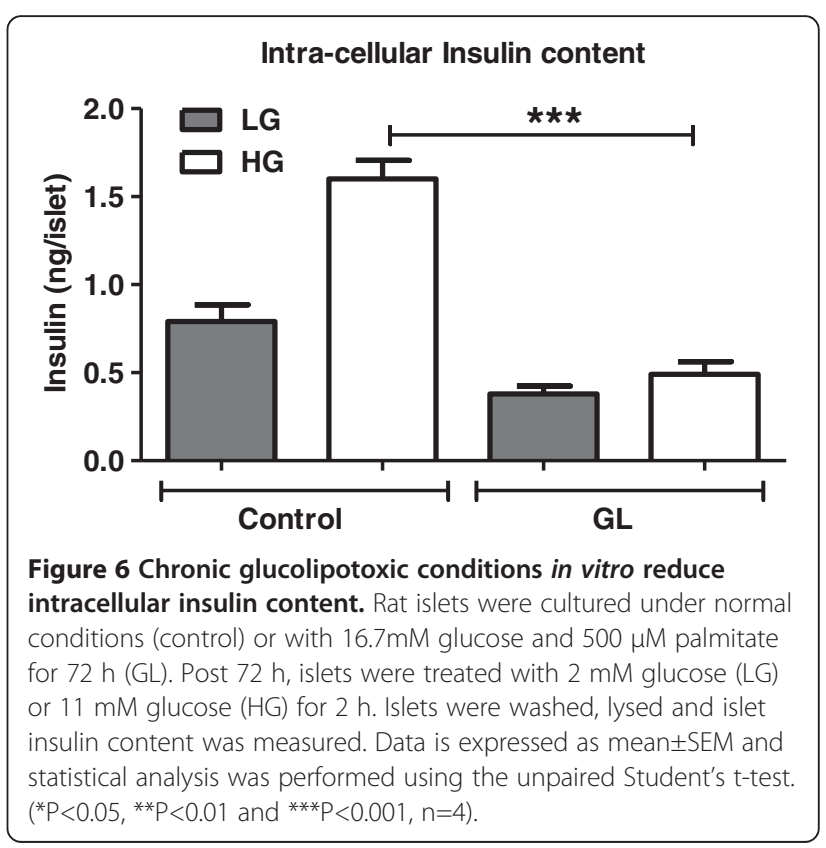



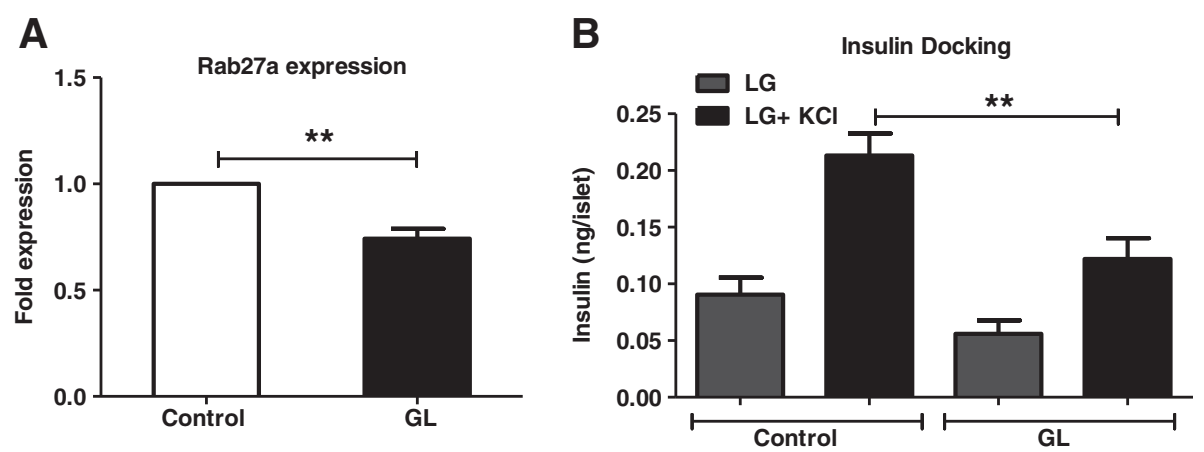

Figure 7 In vitro chronic glucolipotoxicity reduces insulin docking and exocytosis. Rat islets were cultured under normal conditions (control) or with $16.7 \mathrm{mM}$ glucose and $500 \mu \mathrm{M}$ palmitate for $72 \mathrm{~h}$ (GL). Post $72 \mathrm{~h}$, RNA was isolated from islets and the mRNA levels of Rab27a (A) were measured as described in the materials and methods. For measurement of insulin granule docking (B) following incubation under chronic glucolipotoxic conditions, islets were treated with LG (2.5 mM glucose) with or without $30 \mathrm{mM} \mathrm{KCl}$ for 30 min. Secreted insulin was measured as described in the materials and methods. Data are expressed as mean \pm SEM and statistical analysis was performed using the unpaired Student's t-test. ${ }^{*} \mathrm{P}<0.05,{ }^{* *} \mathrm{P}<0.01$ and $\left.{ }^{* * *} \mathrm{P}<0.001, \mathrm{n}=4\right)$.

\section{Discussion}

Despite intensive research, information about the mechanism of action and intracellular signaling pathways activated by glucolipotoxicity remains limited. Such an understanding has clinical relevance since the ability of the beta cell to increase insulin secretion in response to fatty acids is thought to be a predisposing factor for T2DM [25]. In vitro studies have been important to gain a mechanistic understanding of glucolipotoxicity but have not allowed a complete view of glucolipotoxicity-mediated cellular dysregulation due to variations in the concentrations of fatty acids used [24]. This study systematically evaluates specific in vitro glucolipotoxic conditions linking their effect to multiple cellular processes involved in insulin secretion and glucose responsiveness including glucose uptake/metabolism, fatty acid uptake/metabolism, cellular energetics, insulin synthesis, secretion and transport; and calcium dynamics.

We used $16.7 \mathrm{mM}$ glucose and $500 \mu \mathrm{M}$ palmitate in this study after evaluating multiple concentrations for their effect on metabolic stress and cell death (unpublished observations). Under these conditions, we confirmed metabolic stress in pancreatic islets and the NIT1 pancreatic beta cell line as seen by the strong induction of ER stress, oxidative stress and inflammation. As previously reported [27], these conditions also led to cell death as seen by the significant increase in caspase-3 activity (Additional file 2: Figure S2B).

Under chronic glucolipotoxic conditions in vitro, we found that insulin content and GSIS were lowered in rat pancreatic islets. Further, glucose and fat metabolism were impaired in islets correlating with the decrease in mitochondrial number/activity and cellular ATP levels. Chronic glucolipotoxicity reduced cytosolic calcium levels by decreasing calcium mobilization mediated by ITPR.

Based on our findings, we propose a model for the effect of chronic glucolipotoxicity on the pancreatic beta cell (Figure 8). Extended exposure to high glucose and palmitate concentrations leads to a) suppression of glycolysis resulting in reduced cellular ATP and a dampening of the TCA cycle, b) reduction in mitochondrial DNA copy number and activity, c) reduction in PLC-IP3 signaling leading to reduced calcium mobilization, insulin transcription and granule docking, and d) a decrease in insulin transcription and synthesis.

Our in vitro findings recapitulated data from previous glucolipotoxic studies in animal models showing an impact on glucose metabolism, calcium dynamics and insulin secretion/content $[24,44]$. For example, we detected an increase in CD36 expression under glucolipotoxic conditions that correlated with enhanced triglyceride accumulation and reduced GSIS. These findings concur with data from cd36-null mouse models and over-expression studies in INS cell lines $[45,46]$ further validating the in vitro conditions used in our study.

We found it interesting that chronic glucolipotoxic conditions impacted multiple cellular processes including insulin synthesis, content and docking. On the basis of our results, we speculate that chronic glucolipotoxicity impacts insulin content most severely compared to insulin gene transcription, docking and secretion. Future studies will be required to get a more complete understanding of the same.

A key finding in our study is the influence of glucolipotoxicity on mitochondrial number/function. This is in line with the notion that enhanced insulin secretion may require an overall increase in mitochondrial activity/ number as opposed to an isolated increase in an aspect of mitochondrial metabolism $[47,48]$. In this study, we also detected a decrease in insulin granule docking/release under glucolipotoxic conditions indicative of a reduction in the readily releasable pool of insulin, which may have a bearing on the first-phase insulin secretion [43]. Further 


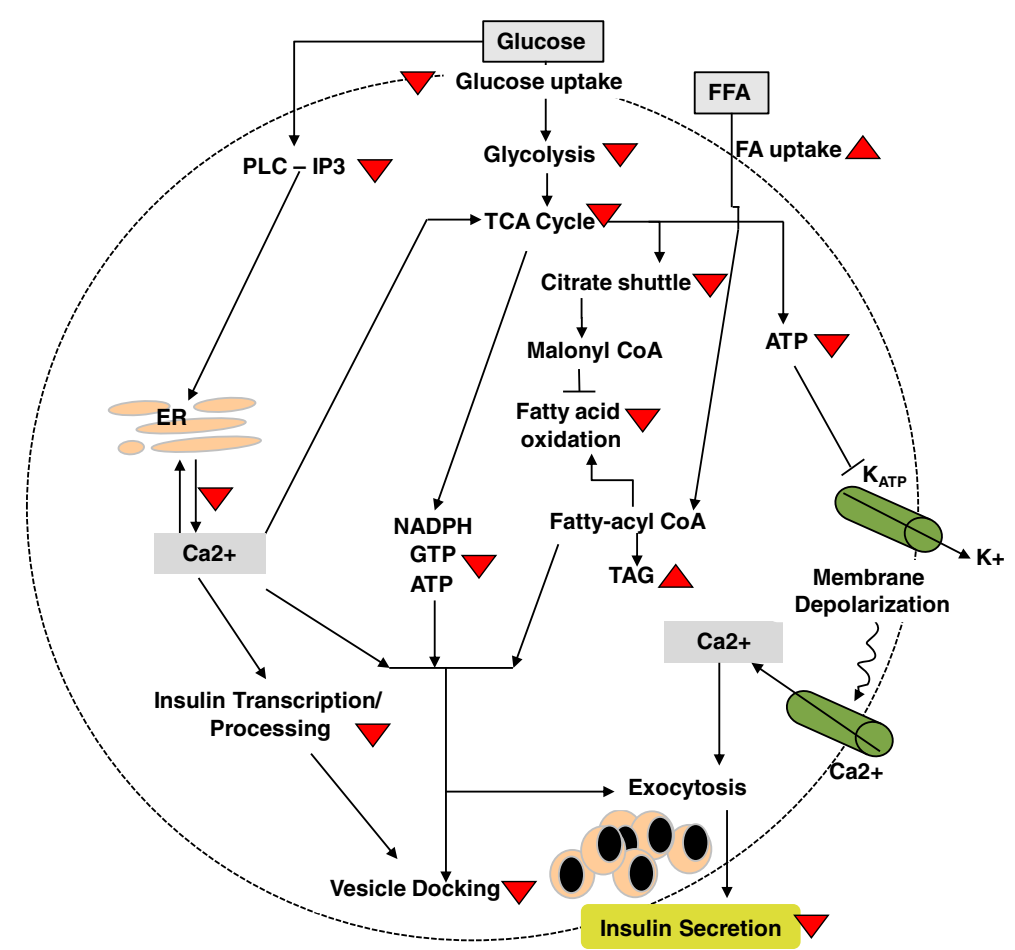

Figure 8 Model for impact of in vitro chronic glucolipotoxicity on cellular processes in pancreatic beta cells. Model describes the signaling pathways involved in glucose and fat metabolism with focus on their coupling to insulin secretion. We found that under chronic glucolipotoxic conditions in vitro, multiple signaling events involved in glucose and fat metabolism are dysregulated leading to impaired insulin synthesis, content and secretion. The effects of chronic glucolipotoxic conditions on the signaling events involved are shown using upward arrowheads (representing an increase) and downward arrowheads (representing a decrease). In the model, arrows indicate activation and blunt arrows indicate inhibition.

studies are required to explore the mechanistic link between docking/exocytosis and the DAG-PKC pathway in more detail.

Increasing insulin secretion is an intensely pursued therapeutic strategy in T2DM. This study yields in vitro assay conditions that can be used to evaluate anti-diabetic agents, specifically insulin secretagogues, currently in development for their impact on glucolipotoxicity-mediated dysregulation. Importantly, an understanding of glucolipotoxicity-mediated cellular dysfunction may yield novel points of therapeutic intervention (i.e., targets/target classes) that hold promise in T2DM treatment. Thus, our study has potential to facilitate an improved understanding of pancreatic beta cell pathophysiology in T2DM.

\section{Conclusions}

Chronic glucolipotoxic conditions comprising high glucose and fatty acid resulted in various defects in key cellular machineries. Glucose sensing machinery involved in uptake and glucose metabolism for insulin secretion was reduced whereas fat uptake and triglyceride storage was increased. Defects in mitochondrial number and activity along with reduced ATP levels were observed under glucolipotoxic conditions. Similarly, beta cells showed increased ER stress, inflammation and apoptosis together with impaired calcium homeostasis. These defects occurred in conjunction with decreased insulin synthesis, insulin vesicle transport, docking and glucose-dependent insulin secretion. Our data provide a first integrated view of beta cell defects across multiple levels under chronic glucolipotoxic conditions.

\section{Methods}

RNA isolation, reverse transcription and quantitative real time polymerase chain reaction (qPCR)

Isolation and preparation of rat islets has been described in detail in Additional file 6 (supplementary methods). All animal studies and protocols were approved by the Institutional Animal Ethics Committee (IAEC) of Connexios Life Sciences Pvt Ltd. Post $72 \mathrm{~h}$ of incubation, total RNA was isolated and $1 \mu \mathrm{g}$ of total RNA was used to generate cDNA (ABI, USA). Gene expression was measured using SYBR Green PCR Master Mix (Eurogenetic, Belgium). Gene primers for Slc2a2/Glut2, Gck, Pc, CD36, PPAR $\alpha$, Pdx1, Ins2, Rab27a, Il1 $\beta$, Nos2a and Actb were based on mRNA sequences from the GenBank nucleotide database and designed in-house. Actb was used as an internal control. The primer sequence for the above gene markers are given in the Additional file 6 (supplementary methods). 


\section{Insulin secretion and content}

Islets were isolated from rats (details in Additional file 6) and cultured in $90 \mathrm{~mm}$ petri-plates with RPMI $11 \mathrm{mM}$ glucose and 10\% FBS and penicillin streptomycin, in the presence or absence of $16.7 \mathrm{mM}$ glucose and $500 \mu \mathrm{M}$ palmitate for $72 \mathrm{~h} / 37^{\circ} \mathrm{C} / 5 \% \mathrm{CO} 2$. Size-matched islets were isolated and transferred into 24-well plates containing $1 \mathrm{ml} \mathrm{KRBH}$ (2.5 mM glucose)/well, and incubated at $37^{\circ} \mathrm{C} / 5 \% \mathrm{CO} 2$ for 1h. After removing the $\mathrm{KRBH}$ buffer, the islets were induced in $\mathrm{KRBH}$ buffer $(250 \mu \mathrm{l} /$ well $)$ at $37^{\circ} \mathrm{C} / 5 \% \mathrm{CO} 2$ for $2 \mathrm{~h}$ at indicated glucose concentrations with/without the specified pharmacological inhibitors. Inhibitors were used at the following concentrations: Radicicol (50 $\mu \mathrm{M}$; Sigma) and Nitrendipine ( $5 \mu \mathrm{M}$; Sigma); 2-APB (10 $\mu \mathrm{M}$, Sigma). Secreted insulin was measured in KRBH buffer using ELISA (Mercodia) as per manufacturer's instructions. To measure insulin secretion in the presence of TCA cycle precursors, islets were prepared as above and treated with $5 \mathrm{mM}$ leucine and $5 \mathrm{mM}$ glutamine-containing $\mathrm{KRBH}$ for $2 \mathrm{~h}$; $2 \mathrm{mM}$ glucose without amino acids was used as a control. Islet lysates were used to measure intracellular insulin content and insulin levels were presented as ng insulin/islet.

\section{Western blotting}

NIT1 (ATCC) cells or rat islets were cultured with $5.5 \mathrm{mM}$ glucose (control) with or without $16.7 \mathrm{mM}$ glucose and $500 \mu \mathrm{M}$ palmitate (GL) for $72 \mathrm{~h}$. After incubation, cells or islets were lysed and total proteins were resolved by SDS-PAGE followed by transfer to nitrocellulose membrane. Protein expression and phosphorylation was measured using

Antibodies against Glut2, CD36 (Abcam), BiP, CHOP, p-eIF2a or $\beta$-actin (Cell Signaling Technology) and HRP conjugated secondary antibody (Bio-Rad). The protein specific signals were detected using chemiluminescence substrate (Pierce) and were quantified using Image-J software (NIH).

\section{Measurement of glucose uptake}

NIT1 cells were cultured with $5.5 \mathrm{mM}$ glucose (control) with or without $16.7 \mathrm{mM}$ glucose and $500 \mu \mathrm{M}$ palmitate (GL) for $72 \mathrm{~h}$. Post $72 \mathrm{~h}$, cells were washed and incubated in glucose-free medium at $37^{\circ} \mathrm{C}$ for $30 \mathrm{~min}$ followed by incubation with $50 \mu \mathrm{M}$ of 2-NBDG (2-(N-(7-Nitrobenz2-oxa-1,3-diazol-4-yl)Amino)-2-Deoxyglucose; Invitrogen) for $15 \mathrm{~min}$. After lysis, 2-NBDG uptake was measured at $465 \mathrm{~nm}$ excitation/540 $\mathrm{nm}$ emission, and normalized to total cellular DNA as measured using bis-benzamide at $360 \mathrm{~nm}$.

\section{Measurement of fatty acid uptake}

NIT1 cells were cultured with $5.5 \mathrm{mM}$ glucose (control) with or without $16.7 \mathrm{mM}$ glucose and $500 \mu \mathrm{M}$ palmitate (GL) for 72 h. Post 72 h, cells were washed and incuba- ted in glucose-free medium at $37^{\circ} \mathrm{C}$ for $30 \mathrm{~min}$ followed by incubation with $1 \mu \mathrm{M}$ of green-fluorescent BODIPY dyes (Molecular Probes) for $10 \mathrm{~min}$. Cells were washed and incubated with $0.4 \%$ trypan blue for $5 \mathrm{~min}$ to quench any excess dye. Subsequently, cells were washed, lysed and BODIPY uptake was measured at $485 \mathrm{~nm}$ excitation/528 nm emission and normalized to total cellular proteins as measured using the Bradford assay (Bio-Rad). BODIPY uptake was represented as \% of uptake under control condition.

\section{Estimation of triglycerides}

NIT1 cells were cultured with $5.5 \mathrm{mM}$ glucose (control) with or without $16.7 \mathrm{mM}$ glucose and $500 \mu \mathrm{M}$ palmitate (GL) for $72 \mathrm{~h}$. After incubation, cells were washed with PBS and lysed. Total cellular protein was estimated using the Bradford assay (Bio-Rad) and triglyceride levels were estimated using an enzymatic assay (DiaSys) as per manufacturer's instructions. TAG levels were normalized to cellular protein levels.

\section{Estimation of mitochondrial DNA copy number}

Freshly isolated rat islets were cultured under control or glucolipotoxic conditions for $72 \mathrm{~h}$. Post $72 \mathrm{~h}$ treatment, islets were harvested in digestion buffer (Tris-10mM, pH8.0, EDTA-1 mM, NaCl-5 mM, SDS-1\% and RNAse-A-10 mg/ $\mathrm{ml}$ ) followed by a phenol-chloroform extraction and ethanol precipitation of total DNA. $5 \mu \mathrm{g}$ DNA was used for quantitative real time PCR. Mitochondrial cytochrome $\mathrm{C}$ oxidase 1 (mtCox1) copy number was measured and normalized to nuclear DNA using hypoxanthine guanine phosphoribosyltransferase (HPRT).

\section{Measurement of islet ATP}

Rat islets were cultured as in the insulin secretion assay. Islets were incubated in the KRBH buffer containing $2.5 \mathrm{mM}$ glucose for $1 \mathrm{~h}$ followed by induction with $11 \mathrm{mM}$ glucose (HG) for $1 \mathrm{~h}$. After $1 \mathrm{~h}$ of incubation, islets were lysed and ATP levels were estimated as per manufacturer's instructions (ATP determination kit, Invitrogen).

\section{Measurement of succinate dehydrogenase activity}

NIT1 cells were cultured in $5.5 \mathrm{mM}$ glucose (control) with or without $16.7 \mathrm{mM}$ glucose and $500 \mu \mathrm{M}$ palmitate (GL) for $72 \mathrm{~h}$. After incubation, cells were washed and incubated in $100 \mathrm{mM}$ potassium phosphate buffer containing $50 \mathrm{mM}$ sucrose, $10 \mathrm{mM}$ sodium azide, $500 \mathrm{mM}$ sodium succinate and $8 \mathrm{mM}$ INT (Iodonitrotetrazolium chloride; Sigma) for $2 \mathrm{~h}$. Cells without sodium succinate were used as a negative control. After $2 \mathrm{~h}$ at $37^{\circ} \mathrm{C}$, INT was dissolved in DMSO and estimated at $644 \mathrm{~nm}$. The difference in absorbance with/ without succinate was calculated, normalized to total cellular protein and represented as \% control SDH activity. 


\section{Estimation of islet IP3}

Freshly isolated rat islets were cultured under normal condition (control) or under glucolipotoxic condition (GL) for $72 \mathrm{~h}$. Islets were then washed and incubated in $\mathrm{KRBH}$ containing $2.5 \mathrm{mM}$ glucose for $1 \mathrm{~h}$ followed by treatment with HG for $5 \mathrm{~min}$. IP3 levels were measured in the lysate using an immunoassay kit (Cusabio IP3 estimation kit).

\section{Estimation of calcium mobilization}

NIT1 cells were cultured with $5.5 \mathrm{mM}$ glucose (control) with or without $16.7 \mathrm{mM}$ glucose and $500 \mu \mathrm{M}$ palmitate (glucolipotoxic) for $72 \mathrm{~h}$. After incubation, cells were washed with calcium-free $\mathrm{KRBH}$ buffer followed by incubation at $37^{\circ} \mathrm{C}$ for $1 \mathrm{~h}$ in Fluo-3-AM calcium indicator fluorescent dye (Invitrogen). Cells were then induced with either low $(2.5 \mathrm{mM})$ or high $(16.7 \mathrm{mM})$ glucose and the fluorescence was measured at $485 \mathrm{~nm}$. The baseline reading was established by reading fluorescence for $1 \mathrm{mi}$ nute (from $\mathrm{t}=0$ to $\mathrm{t}=1$ minute) at $6 \mathrm{~s}$ intervals. The indicated glucose concentrations were added at $\mathrm{t}=1$ minute. After mixing for $5 \mathrm{~s}$, the final reading was taken for $3 \mathrm{mi}-$ nutes (from $\mathrm{t}=1$ minute to $\mathrm{t}=4 \mathrm{~min}$ ) at $6 \mathrm{~s}$ intervals.

\section{Exocytosis of docked insulin granules}

Islets were cultured as in the insulin secretion assay, washed and incubated in KRBH buffer containing $2.5 \mathrm{mM}$ glucose for $1 \mathrm{~h}$. Islets were treated with low glucose alone (2 $\mathrm{mM}$ ) with/without $30 \mathrm{mM} \mathrm{KCl}$ in $\mathrm{KRBH}$ for $30 \mathrm{~min}$ followed by estimation of secreted insulin in the buffer.

\section{Statistical methods}

Data are expressed as mean \pm SEM and significance was calculated using the unpaired Student's t-test. * indicates $\mathrm{p}<0.05$; $*$ indicates $\mathrm{p}<0.01$; ${ }^{* * *}$ indicates $\mathrm{p}<0.001$ compared to the respective control. Unless mentioned otherwise, $n=4$ across experiments. Miscrosoft Excel was used for statistical analyses.

\section{Additional files}

Additional file 1: Figure S1. Chronic glucolipotoxic conditions increase inflammatory cytokines gene expression and cellular stress in pancreatic $\beta$-cells. Rat islets or NIT1 cells were cultured under normal conditions (control) or with $16.7 \mathrm{mM}$ glucose and $500 \mu \mathrm{M}$ palmitate for $72 \mathrm{~h}$ (GL). Post $72 \mathrm{~h}$, RNA was isolated from islets and the mRNA levels of IL $1 \mathrm{~b}$ (A) and Nos2a (B) were measured as described in the materials and methods. Both ROS (C) and nitric oxide (D) levels were estimated in NIT1 cells as described in the Additional file 6. Data are expressed as mean \pm SEM and statistical analysis was performed using the unpaired Student's t-test. $\left({ }^{*} P<0.05,{ }^{* * P}<0.01\right.$ and $\left.{ }^{* *} P<0.001, n=4\right)$.

Additional file 2: Figure S2. Chronic glucolipotoxic conditions increase endoplasmic reticulum stress and apoptosis in pancreatic $\beta$-cells. NIT1 cells were cultured under normal conditions (control) or with $16.7 \mathrm{mM}$ glucose and $500 \mu \mathrm{M}$ palmitate for $72 \mathrm{~h}$ (GL). Post $72 \mathrm{~h}$, changes in ER stress markers (A) were analyzed using anti-CHOP, anti-BiP and phosphospecific EIF2a antibodies; $\beta$-actin was used as the internal control. Both Caspase-3 activity (B) and cell viability (MTT assay) were measured in
NIT1 cells as described in the Additional file 6. Data are expressed as mean \pm SEM and statistical analysis was performed using the unpaired Student's t-test. ( ${ }^{*} P<0.05$, ${ }^{*} P<0.01$ and $\left.{ }^{* *} P<0.001, n=4\right)$.

Additional file 3: Figure S3. Glucose metabolism is impaired under glucolipotoxic condition. NIT1 cells were cultured under normal conditions (control) or with $16.7 \mathrm{mM}$ glucose and 500 $\mu \mathrm{M}$ palmitate for $72 \mathrm{~h}(\mathrm{GL})$. Post $72 \mathrm{~h}$, cells were lysed and cellular NADPH levels were measured (A) and culture medium was used for the estimation of lactate levels (B). Data are expressed as mean \pm SEM and statistical analysis was performed using the unpaired Student's t-test. ( ${ }^{*} P<0.05$, ${ }^{*} P<0.01$ and ***P $<0.001, n=3-4$ ).

Additional file 4: Figure S4. Cytoplasmic calcium increase is required for insulin secretion under glucolipotoxic condition. Rat islets were cultured under normal conditions (control) or with $16.7 \mathrm{mM}$ glucose and $500 \mu \mathrm{M}$ palmitate for $72 \mathrm{~h}$ (GL). Post 72 h, islets were treated with $11 \mathrm{mM}$ glucose $(\mathrm{HG}$ ) alone or with inhibitors (5 $\mu \mathrm{M}$ Nitrendipine and $10 \mu \mathrm{M}$ 2-APB) for $2 \mathrm{~h}$ and secreted insulin was measured as described in the materials and methods. Data are expressed as mean \pm SEM and statistical analysis was performed using the unpaired Student's t-test. ( ${ }^{*} \mathrm{P}<0.05$, ${ }^{* *} \mathrm{P}<0.01$ and $\left.{ }^{* *} \mathrm{P}<0.001, \mathrm{n}=4\right)$.

Additional file 5: Figure S5. Chronic GL conditions reduce insulin synthesis and vesicle transport. Rat islets or NIT1 cells were cultured under normal conditions (control) or with $16.7 \mathrm{mM}$ glucose and $500 \mu \mathrm{M}$ palmitate for $72 \mathrm{~h}(\mathrm{GL})$. Post $72 \mathrm{~h}$, RNA was isolated from both islets and cells. The mRNA levels of PDX1 (A) and Insulin (B) were measured in islets and Rab27a levels (C) in cells were measured as described in the materials and methods. Data are expressed as mean \pm SEM and statistical analysis was performed using the unpaired Student's t-test. $\left({ }^{*} P<0.05\right.$, ${ }^{* *} \mathrm{P}<0.01$ and $\left.{ }^{* *} \mathrm{P}<0.001, \mathrm{n}=4\right)$.

Additional file 6: Supplementary methods.

\section{Competing interest}

The authors do not declare any competing interest.

\section{Authors' contributions}

BS, RA, YAN, PPM, SN, PCS, RS, KN, SMK and VMK carried out experiments; VMK, SBP and JMR planned/executed the study and analyzed data. NU, MOA, VMK, SMK, SBP and JMR analyzed data and contributed to writing the paper. All authors read and approved the final manuscript.

\section{Acknowledgements}

We would like to thank all the members of the in vitro experimentation group with specific mention of Bhawna Chandravanshi, Niketa Pawar, Aishwarya Sathyanarayan and Jayalaxmi Shetty at Connexios Life Sciences Pvt Ltd. for their assistance in the study. We would like to thank Dr.Lokesh Joshi, Dr. Raghavendra Rao, Dr. Mathiyazhagan and Aseem Premnath for their critical review of the manuscript and Dr. Rohit Kulkarni for helpful discussions.

\section{Funding}

These studies were supported by Connexios Life Sciences Pvt Ltd., a Nadathur Holdings company.

Received: 17 October 2012 Accepted: 24 June 2013

Published: 1 July 2013

\section{References}

1. Prentki M, Nolan CJ: Islet beta cell failure in type 2 diabetes. $J$ Clin Invest 2006, 116:1802-1812.

2. Defronzo RA: Banting Lecture. From the triumvirate to the ominous octet: a new paradigm for the treatment of type 2 diabetes mellitus. Diabetes 2009, 58:773-795.

3. Poitout V, Amyot J, Semache M, Zarrouki B, Hagman D, Fontés G: Glucolipotoxicity of the pancreatic beta cell. Biochim Biophys Acta 1801, 2010:289-298.

4. Poitout V, Robertson RP: Secondary beta-cell failure in type 2 diabetes-a convergence of glucotoxicity and lipotoxicity. Endocrinology 2002, 143:339-342. 
5. El-Assaad W, Buteau J, Peyot ML, Nolan C, Roduit R, Hardy S, Joly E, Dbaibo G, Rosenberg L, Prentki M: Saturated fatty acids synergize with elevated glucose to cause pancreatic beta-cell death. Endocrino/ 2003, 144:4154-4163.

6. Jacqueminet S, Briaud I, Rouault C, Reach G, Poitout V: Inhibition of insulin gene expression by long-term exposure of pancreatic beta cells to palmitate is dependent on the presence of a stimulatory glucose concentration. Metabolism 2000, 49:532-536.

7. Shimabukuro M, Zhou YT, Levi M, Unger RH: Fatty acid-induced beta cell apoptosis: a link between obesity and diabetes. Proc Natl Acad Sci USA 1998, 95:2498-2502.

8. Zhou YP, Grill VE: Long-term exposure of rat pancreatic islets to fatty acids inhibits glucose-induced insulin secretion and biosynthesis through a glucose fatty acid cycle. J Clin Invest 1994, 93:870-876.

9. Henquin JC: Triggering and amplifying pathways of regulation of insulin secretion by glucose. Diabetes 2000, 49:1751-1760.

10. Farfari S, Schulz V, Corkey B, Prentki M: Glucose-regulated anaplerosis and cataplerosis in pancreatic beta-cells: possible implication of a pyruvate/ citrate shuttle in insulin secretion. Diabetes 2000, 49:718-726.

11. Deeney JT, Gromada J, Høy M, Olsen HL, Rhodes CJ, Prentki M, Berggren PO, Corkey BE: Acute stimulation with long chain acyl-CoA enhances exocytosis in insulin-secreting cells HIT T-15 and NMRI beta-cells. J Biol Chem 2000, 275:9363-9368

12. Prentki M, Vischer S, Glennon MC, Regazzi R, Deeney JT, Corkey BE: MalonylCoA and long chain acyl-CoA esters as metabolic coupling factors in nutrient-induced insulin secretion. J Bio/ Chem 1992, 267:5802-5810.

13. Ashcroft FM, Gribble FM: ATP-sensitive $\mathrm{K}+$ channels and insulin secretion: their role in health and disease. Diabetologia 1999, 42:903-919.

14. Zawalich WS, Zawalich KC, Kelley GG: Regulation of insulin release by phospholipase $C$ activation in mouse islets: differential effects of glucose and neurohumoral stimulation. Endocrinol 1995, 136:4903-4909.

15. Persaud SJ, Jones PM, Howell SL: Activation of PKC is essential for sustained insulin secretion in response to cholinergic stimulation Biochim Biophys Acta 1991, 1091:120-122.

16. Uchida T, Iwashita N, Ohara-Imaizumi M, Ogihara T, Nagai S, Choi JB, Tamura Y, Tada N, Kawamori R, Nakayama Kl, Nagamatsu S, Watada H: Protein kinase $C$ delta plays a non-redundant role in insulin secretion in pancreatic beta cells. J Biol Chem 2007, 282:2707-2716.

17. Dyachok O, Idevall-Hagren O, Sagetorp J, Tian G, Wuttke A, Arrieumerlou C, Akusjärvi G, Gylfe E, Tengholm A: Glucose-induced cyclic AMP oscillations regulate pulsatile insulin secretion. Cell Metab 2008, 8:26-37.

18. Koya D, King GL: Protein kinase $C$ activation and the development of diabetic complications. Diabetes 1998, 47:859-866.

19. Hagman DK, Latour MG, Chakrabarti SK, Fontes G, Amyot J, Tremblay C, Semache M, Lausier JA, Roskens V, et al: Cyclical and alternating infusions of glucose and intralipid in rats inhibit insulin gene expression and Pdx1 binding in islets. Diabetes 2008, 57:424-431.

20. Hou JC, Min L, Pessin JE: Insulin granule biogenesis, trafficking and exocytosis. Vitam Horm 2009, 80:473-506.

21. Wang Z, Thurmond DC: Mechanisms of biphasic insulin-granule exocytosis - roles of the cytoskeleton, small GTPases and SNARE proteins. J Cell Sci 2009, 122:893-903.

22. Unger RH, Grundy S: Hyperglycaemia as an inducer as well as a consequence of impaired islet cell function and insulin resistance: implications for the management of diabetes. Diabetologia 1985, 28:119-121.

23. Unger RH: Lipotoxicity in the pathogenesis of obesity-dependent NIDDM. Genetic and clinical implications. Diabetes 1995, 44:863-870.

24. Poitout V: Glucolipotoxicity of the pancreatic beta-cell: myth or reality? Biochem Soc Trans 2008, 36:901-904.

25. Kashyap S, Belfort R, Gastaldelli A, Pratipanawatr T, Berria R, Pratipanawatr W, Bajaj $M$, Mandarino L, DeFronzo R, Cusi $K$ : A sustained increase in plasma free fatty acids impairs insulin secretion in nondiabetic subjects genetically predisposed to develop type 2 diabetes. Diabetes 2003, 52:2461-2474.

26. Poitout V, Robertson RP: Glucolipotoxicity: fuel excess and beta-cell dysfunction. Endocr Rev 2008, 29:351-366

27. El-Assaad W, Joly E, Barbeau A, Sladek R, Buteau J, Maestre I, Pepin E, Zhao S, Iglesias J, et al: Glucolipotoxicity alters lipid partitioning and causes mitochondrial dysfunction, cholesterol, and ceramide deposition and reactive oxygen species production in INS832/13 ß-cells. Endocrinology 2010, 151:3061-3073

28. Abaraviciene SM, Lundquist I, Salehi A: Rosiglitazone counteracts palmitate-induced beta-cell dysfunction by suppression of MAP kinase, inducible nitric oxide synthase and caspase 3 activities. Cell Mol Life Sci 2008, 65:2256-2265.

29. Tanabe K, Liu Y, Hasan SD, Martinez SC, Cras-Méneur C, Welling CM, BernalMizrachi E, Tanizawa Y, Rhodes CJ, Zmuda E, et al: Glucose and fatty acids synergize to promote B-cell apoptosis through activation of glycogen synthase kinase $3 \beta$ independent of JNK activation. PLoS One 2011, 6:e18146.

30. Gremlich S, Bonny C, Waeber G, Thorens B: Fatty acids decrease IDX-1 expression in rat pancreatic islets and reduce GLUT2, glucokinase, insulin, and somatostatin levels. J Biol Chem 1997, 272:30261-30269.

31. Matschinsky FM, Glaser B, Magnuson MA: Pancreatic beta-cell glucokinase: closing the gap between theoretical concepts and experimental realities. Diabetes 1998, 47:307-315

32. Han J, Liu YQ: Reduction of islet pyruvate carboxylase activity might be related to the development of type 2 diabetes mellitus in Agouti-K mice. J Endocrinol 2010, 204:143-152.

33. Marshall C, Hitman GA, Cassell PG, Turner MD: Effect of glucolipotoxicity and rosiglitazone upon insulin secretion. Biochem Biophys Res Commun 2007, 356:756-762

34. Guay C, Madiraju SR, Aumais A, Joly E, Prentki M: A role for ATP-citrate lyase, malic enzyme, and pyruvate/citrate cycling in glucose-induced insulin secretion. J Biol Chem 2007, 282:35657-35665.

35. Poitout $\mathrm{V}$ : The ins and outs of fatty acids on the pancreatic beta cell. Trends Endocrinol Metab 2003, 14:201-203.

36. Broca C, Brennan L, Petit P, Newsholme P, Maechler P: Mitochondriaderived glutamate at the interplay between branched-chain amino acid and glucose-induced insulin secretion. FEBS Lett 2003, 545:167-172.

37. Plant TD: Properties and calcium-dependent inactivation of calcium currents in cultured mouse pancreatic B-cells. J Physiol 1988, 404:731-747.

38. Maruyama T, Kanaji T, Nakade S, Kanno T, Mikoshiba K: 2APB, 2aminoethoxydiphenyl borate, a membrane-penetrable modulator of Ins (1,4,5)P3-induced Ca2+ release. J Biochem 1997, 122:498-505.

39. Tian $Y$, Laychock SG: Protein Kinase $C$ and Calcium Regulation of Adenylyl Cyclase in Isolated Rat Pancreatic Islets. Diabetes 2001, 50:2505-2513.

40. Roger B, Papin J, Vacher P, Raoux M, Mulot A, Dubois M, Kerr-Conte J, Voy $\mathrm{BH}$, Pattou F, et al: Adenylyl cyclase 8 is central to glucagon-like peptide 1 signalling and effects of chronically elevated glucose in rat and human pancreatic beta cells. Diabetologia 2011, 54:390-402.

41. Kato T, Shimano H, Yamamoto T, Yokoo T, Endo Y, Ishikawa M, Matsuzaka T, Nakagawa Y, Kumadaki S, et al: Granuphilin is activated by SREBP-1c and involved in impaired insulin secretion in diabetic mice. Cell Metab 2006, 4:143-154.

42. Olofsson CS, Collins S, Bengtsson M, Eliasson L, Salehi A, Shimomura K, Tarasov A, Holm C, Ashcroft F, Rorsman P: Long-term exposure to glucose and lipids inhibits glucose-induced insulin secretion downstream of granule fusion with plasma membrane. Diabetes 2007, 56:1888-1897.

43. Daniel S, Noda M, Straub SG, Sharp GW: Identification of the docked granule pool responsible for the first phase of glucose-stimulated insulin secretion. Diabetes 1999, 48:1686-1690.

44. Gwiazda KS, Yang TL, Lin Y, Johnson JD: Effects of palmitate on ER and cytosolic Ca2+ homeostasis in beta-cells. Am J Physiol Endocrinol Metab 2009, 296:E690-E701.

45. Hajri T, Han XX, Bonen A, Abumrad NA: Defective fatty acid uptake modulates insulin responsiveness and metabolic responses to diet in CD36-null mice. J Clin Invest 2002, 109:1381-1389.

46. Wallin $T$, Ma Z, Ogata $H$, Jørgensen $H_{\text {, lezzi } M}$, Wang $H$, Wollheim $C B$, Björklund A: Facilitation of fatty acid uptake by $C D 36$ in insulin-producing cells reduces fatty-acid-induced insulin secretion and glucose regulation of fatty acid oxidation. Biochim Biophys Acta 1801, 2010:191-197.

47. Maechler $P$, Wollheim CB: Mitochondrial signals in glucose-stimulated insulin secretion in the beta cell. J Physiol 2000, 529:49-56.

48. Jang YO, Quan X, Das R, Xu S, Chung CH, Ahn CM, Baik SK, Kong ID, Park KS, Kim MY: High-dose clevudine impairs mitochondrial function and glucosestimulated insulin secretion in INS-1E cells. BMC Gastroenterol 2012, 12:4.

doi:10.1186/1471-2121-14-31

Cite this article as: Somesh et al.: Chronic glucolipotoxic conditions in pancreatic islets impair insulin secretion due to dysregulated calcium dynamics, glucose responsiveness and mitochondrial activity. BMC Cell Biology 2013 14:31. 